\title{
Risk of Psychiatric Problems for People with Negative Psychosocial Impacts in Compared to Positive Attitude during Covid-19 Pandemic: A Systematic Review
}

\author{
Yuliana* \\ Anatomy Department, Udayana University, Indonesia \\ *Corresponding Author. Email: yuliana@unud.ac.id
}

\begin{abstract}
Introduction: COVID-19 pandemic has negative or positive psychosocial impacts on society. Fear, uncertainty, insomnia, depression, anxiety, and suicide are the negative psychosocial impacts. These psychosocial impacts may cause psychiatric problems. This paper aims to describe the risk of psychiatric problems for people with negative psychosocial impacts in compared to positive attitudes during COVID-19 pandemic.

Methods : This systematic literature review used these keywords: 'fear', 'uncertainty', 'COVID19', 'psychiatric problem' and 'society'. The search engines used were PubMed and Science Direct. There were 302 papers from PubMed and 134 papers from Science Direct at the beginning. To ascertain the quality of the selection, the process was done twice. Narrative reviews were excluded. Finally, 19 manuscripts were selected for review. PRISMA guidelines were used in the reviewing procedures.

Results: From 18 cross-sectional studies and one randomized control trial, it was found that females and the elderly are prone to anxiety and depression. Other risk factors are lowincome, sign of infection, previous contact history with COVID-19 patients, too much information about COVID-19, and pseudoscientific beliefs. Positive protective factors are good self-talk skills, coping strategy mechanisms, compliance with the rules, and a high level of mental resilience.

Conclusion: Anxiety and depression prevention related to Covid-19 can be done through strengthening the positive protective factor and minimize the risk factors. COVID-19 pandemic should be seen as an opportunity to strengthen positive mental attitudes.
\end{abstract}

Keywords: COVID-19, Psychosocial Impacts, Society, psychiatric problems

Article History: Received: 23 ${ }^{\text {th }}$ April 2021, Revised: 29th June 2021, Accepted: $12^{\text {th }}$ July 2021

\section{Introduction}

The COVID-19 pandemic has psychosocial impacts on society. The psychosocial impacts can be negative or positive. All depends on the characteristics, adjustment, and resilience in facing this new condition. 1 Fear, uncertainty, confusion, insomnia, depression, anxiety, and suicide are the symptoms of the negative psychosocial impacts. Positive psychosocial impacts can be noveltyseeking behavior, new cognitive behavior (new skill mastery), and new positive activities (indoor exercise). ${ }^{2}$

In a study of 8,806 adults from eight countries/regions, it was found that there were some factors related positively to a probable generalized anxiety disorder 
(GAD) and major depressive episode (MDE). Those factors were a weak sense of coherence, isolation, wrong beliefs, younger age, threat perception (for oneself, family, country, or world), authorities mistrust, stigma, financial problem, and too much information about COVID-19. Positive psychosocial impacts can be attained when those factors are minimal. Too much information about COVID-19 can lead to overload and distress. ${ }^{2}$

Psychosocial impacts affect attention and decision-making ability. Too much information can easily spread positive and negative psychosocial impacts. The reaction depends on the ability to understand and integrate. It is important to identify positive and negative factors contributing to various psychosocial impacts of the individuals. Sociodemographic and sociocultural are the determinant factors. ${ }^{1}$

Lockdown has forced people to think out of the box. Novelty-seeking might increase the creativity to solve problems. Adjustment to unfamiliar stimuli may increase creativity. The emotional reaction may vary. A longitudinal study in Chinese University Students for six months revealed that stress, anxiety, and depression were decreased by novelty seeking. Researchers did measurement three times, i.e. before the COVID-19 began, during the lockdown, and after the lockdown was over. The results showed that novelty-seeking decreases anxiety, depression, and stress. ${ }^{1}$

The meaning maintenance model consists of understanding that human beings make meaning and find associations in any situation. When expectations and existing conditions are meaningless, there will be psychological distress. The COVID-19 pandemic disrupts expectations. This might motivate noveltyseeking behavior to maintain expectations and meaningful life. Coherent relations can maintain good mental health. Creativity can help people in restructuring new meaning and removing boredom. It is beneficial in improving mental health. Useful new positive activities are indoor exercises (yoga), music and art, and new skill mastery (cooking, gardening, and online video making). ${ }^{1,2}$
The meaning maintenance model has four domains. They are self-esteem, belongingness, certainty, and symbolic immortality. Creative activities can be done to reach those domains. A novel idea, product, cognitive activity, or solution are the answers for those activities. Important characteristics to tackle difficult situations are adaptive, flexible, autonomous, dynamic, open, playful, humorous, divergent thinking, and curious (novelty seeking) in a new situation. Noveltyseeking behavior is related to neurotransmitter and risk-taking activity. Multiple perspectives point of view must be implemented to have a new positive appraisal of any situation. Stress and anxiety during isolation can be minimized by exploring a new idea and finding the positive meaning of any situation. ${ }^{1}$ Extend capacities and learn new things are developed by novelty-seeking behavior. The positive emotional state is developed through positive novelty-seeking behavior. ${ }^{2}$

The seeking system comes from the medial forebrain bundle, which is helping someone to have social contact through playing and interaction. Stimulating these areas creates positive emotions and behavior. COVID-19 pandemic forces people not to do activities outdoor. It causes sadness, anger, and fear. ${ }^{3}$ Fear is associated with depression and anxiety. ${ }^{4}$ Those negative feelings are easily spread.

Although the vaccine has been developed, there will be new problems with the vaccine and the pandemic itself. This paper aims to describe the psychosocial impacts of the COVID-19 pandemic in society.

\section{Methods}

Eligibility criteria

Criteria are created based on the PICO framework. PICO criteria can be seen in Table 1. 
Table 1. PICO Criteria of the Study

\begin{tabular}{ll} 
Patient/population & $\begin{array}{l}\text { Communities } \\
\text { with negative } \\
\text { psychosocial } \\
\text { impacts }\end{array}$ \\
\hline Intervention & $\begin{array}{l}\text { COVID-19 } \\
\text { pandemic }\end{array}$ \\
\hline Comparator & Positive attitude \\
\hline Outcome & $\begin{array}{l}\text { Risks of } \\
\text { psychiatric } \\
\text { problems }\end{array}$ \\
\hline
\end{tabular}

Type of studies

This review included full-text studies in English comparing negative and positive psychosocial impacts during the COVID-19 pandemic. Case reports, letters to the editor, and narrative review were excluded.

\section{Participants}

This review included studies with patients of all ages and gender who filled any questionnaire about anxiety, depression, fear, uncertainty, or any psychiatric problems.

\section{Interventions}

The intervention was COVID-19 pandemic.

\section{Outcomes}

The results investigated in this review were psychiatric problems such as depression, anxiety, suicide, schizophrenia, etc.

\section{Information sources}

We used keywords using the Boolean operator. In this study, we used keywords ("fear" OR "uncertainty" OR "psychiatric problem") AND (society) AND (COVID-19) in PubMed and Science direct databases as search engines to find suitable journals

\section{Study selection}

The study selection began with the removal of duplicate records. The irrelevant studies were excluded by screening the titles and abstracts. Firstly, title and abstract must contain at least COVID-19 keywords with one of the other keywords such as fear or uncertainty or psychiatric problem, or describing about negative and/or positive psychosocial impacts during the COVID-19 pandemic. After that, if the free full text is available in English or Indonesian, then the reading and selection will be continued. Otherwise, it will be removed. Full text screening steps are conducted by reading the abstract and conclusion and examining the type of the text. Case reports, letters to the editor, and narrative reviews were excluded.

\section{Results}

\section{Study Selection}

Based on the initial search strategy, there were 302 papers from PubMed and 134 papers from Science Direct found. By the title and abstract screening, 399 articles were excluded, which left with 37 relevant studies. Studies that didn't provide all the information needed were excluded. Finally, there were 19 articles included in the review. PRISMA study flow diagram is described in Figure 1.

\section{Study Characteristics}

We included 19 full-text articles which are 18 cross-sectional (prevalence) studies and 1 Randomized Control Trial (RCT). The articles were published in 2020 and 2021. The Joanna Briggs Institute (JBI) score was used to analyze the quality of the studies. The results are provided in Table 2.

There are some negative psychosocial impacts for some vulnerable groups. Besides that, there are also positive attitudes and better characteristics for lowering the risk of psychiatric problems. From 19 studies, most of them used questionnaire about negative psychosocial impacts such as fear, anxiety, stress, uncertainty, and depression. One study used the stigma discrimination and fear scale.

They are negative psychosocial impacts. However, there are four studies used the combination of positive psychosocial impact such as coping strategy and anxiety score as in a study which was conducted by Song et al., study on combination between self-talk questionnaires and coping strategies questionnaires was conducted by Darmichi et. al., combination of COVID-19 fear scale 


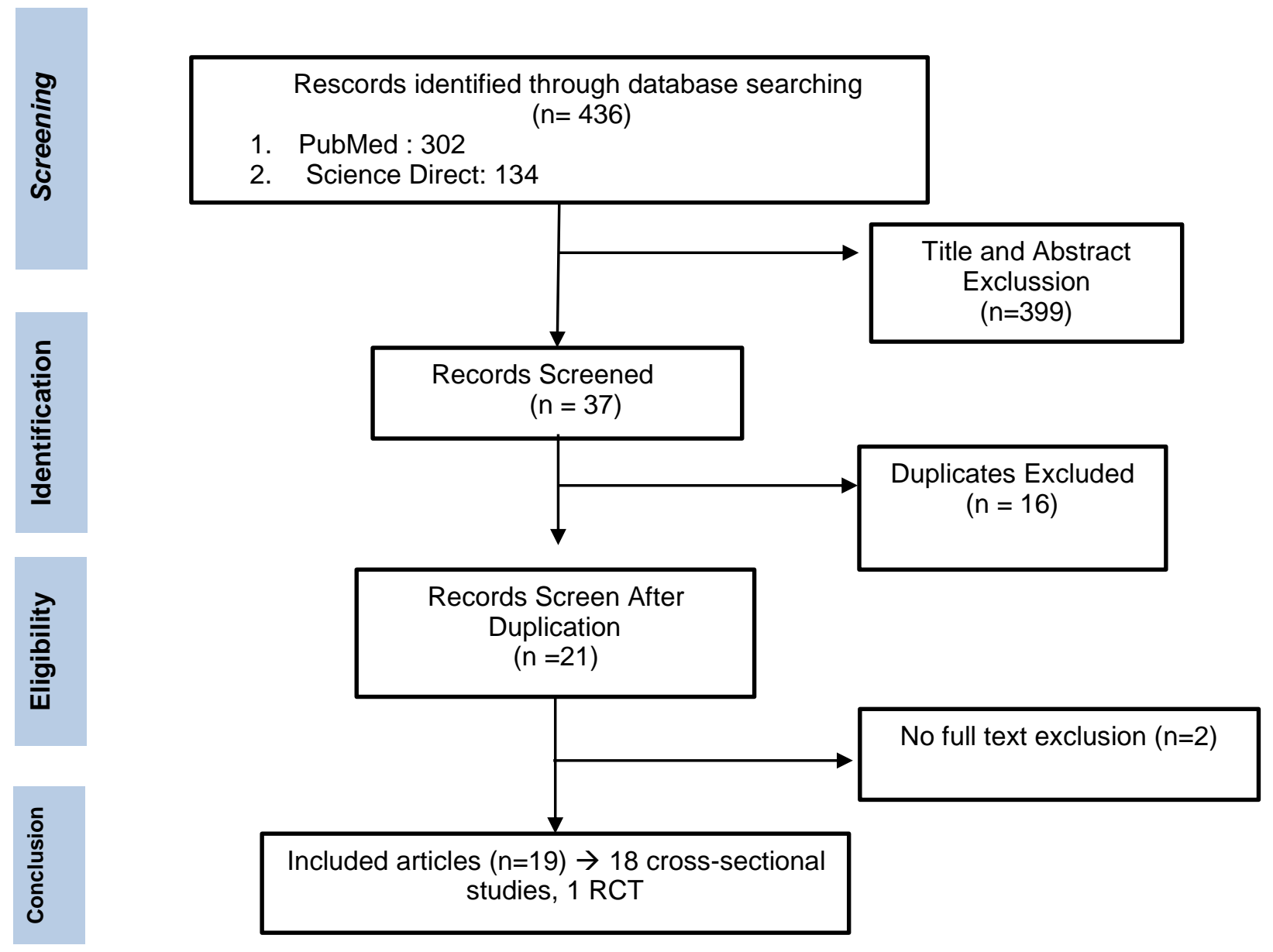

Figure 1. PRISMA Flow Diagram

and health questionnaire by Liang et al, and combination of the fear scale, uncertainty, depression and stress scale with the positivity scale. One study by Gascon et al in Spain used the pseudoscientific belief score.

\section{Discussion}

COVID-19 pandemic must be faced and handled with positive thoughts. The strength of those studies was the insights about the positive and negative psychosocial impacts about the COVID-19 pandemic. This paper has limitation for not providing a metaanalysis. Therefore, a meta-analysis to comprehend the positive and negative psychosocial impacts of covid19 should be done. To counter the negative psychosocial impacts, the negative risk factors such as psychosocial must be combined with the positive attitudes. Below are some categorizations of negative and positive risk factors in handling the COVID-19 pandemic.

\section{Negative risk factors}

Negative attitudes based on included studies are less knowledge about COVID-19 and feeling uncertain about winning and controlling COVID-19 infection. ${ }^{5}$ Schnell and Krampe found that lockdown and crisis of meaningfulness lead to the risk for anxiety and depression. They also assessed the Knowledge, Attitude, and Practice for the COVID-19. ${ }^{6}$

Less favorable demographic characteristics are elderly, obese, and having chronic diseases. ${ }^{5}$ Studies about COVID-19 infection revealed that patients with comorbidities and the elderly had a higher mortality rate than the younger ones. This might be the reason why the depression and anxiety scores were higher among the elderly. ${ }^{11}$ Female is more prone to anxiety based on an online questionnaire. ${ }^{14,15,18,21,22}$ Overuse phone for COVID-19 news searching gave more negative impacts. 
Table 2. Characteristic, JBI score, measurement, and outcome of Inclusion Studies

\begin{tabular}{|c|c|c|c|c|c|}
\hline No & $\begin{array}{l}\text { Author, year, } \\
\text { place, study } \\
\text { design, JBI score }\end{array}$ & $\begin{array}{l}\text { Number of } \\
\text { participants, } \\
\text { demographic } \\
\text { characteristic }\end{array}$ & $\begin{array}{l}\text { Risk of psychiatric } \\
\text { problems } \\
\text { measurement (score) }\end{array}$ & $\begin{array}{l}\text { Negative psychosocial } \\
\text { impacts/attitude/less favorable } \\
\text { demographic characteristics }\end{array}$ & $\begin{array}{l}\text { Positive protective } \\
\text { factors / } \\
\text { attitude/favorable } \\
\text { demographic } \\
\text { characteristics }\end{array}$ \\
\hline 1 & $\begin{array}{l}\text { Rias et al., } 2020 \\
\text { Indonesia, } \\
\text { cross-sectional, } \\
\text { prevalence study. }{ }^{5} \\
\text { JBI Score } 8 / 9\end{array}$ & $\begin{array}{l}1,082 \\
\text { participants } \\
(61.1 \% \text { female })\end{array}$ & $\begin{array}{l}\text { Depression, Anxiety, } \\
\text { and Stress Scale } \\
\text { Daily Spiritual } \\
\text { Experiences Scale, } \\
\text { KAP (Knowledge, } \\
\text { Attitude, and Practice)- } \\
\text { COVID-19 } \\
\text { questionnaire. }\end{array}$ & $\begin{array}{l}\text { Less knowledge about COVID-19, } \\
\text { elderly, obese, having chronic } \\
\text { disease, uncertainty about winning } \\
\text { and controlling COVID-19 infection }\end{array}$ & $\begin{array}{l}\text { Spirituality increases } \\
\text { self-control, decreases } \\
\text { anxiety score, good } \\
\text { knowledge about } \\
\text { COVID-19, single, } \\
\text { young }\end{array}$ \\
\hline 2 & $\begin{array}{l}\text { Schnell and Krampe, } \\
2020 \\
\text { Germany, Austria, } \\
\text { cross-sectional } \\
\text { prevalence study. }{ }^{6} \\
\text { JBI score } 8 / 9\end{array}$ & $\begin{array}{l}1,538 \\
\text { participants } \\
(64.5 \% \text { female })\end{array}$ & $\begin{array}{l}\text { Acute COVID-19 } \\
\text { stress, meaningfulness } \\
\text { and crisis of meaning } \\
\text { (SoMe), self-control } \\
\text { Depression and anxiety } \\
\text { (PHQ-4) score }\end{array}$ & $\begin{array}{ll}\text { Lockdown, } & \text { crisis } \\
\text { meaningfulness } & \end{array}$ & $\begin{array}{l}\text { Meaningfulness, self- } \\
\text { control, less acute } \\
\text { stress for older age, } \\
\text { married person, living } \\
\text { in a house, active } \\
\text { working }\end{array}$ \\
\hline 3 & $\begin{array}{l}\text { Genereux et al., } \\
2020, \quad \text { Canada, } \\
\text { United States, } \\
\text { England, } \\
\text { Switzerland, } \\
\text { Belgium, Hong } \\
\text { Kong, Philippines, } \\
\text { New Zealand,cross- } \\
\text { sectional, } \\
\text { prevalence study. }{ }^{2} \\
\text { JBl score } 7 / 9\end{array}$ & $\begin{array}{l}8,806 \\
\text { participants } \\
\text { (51.9\% female) }\end{array}$ & $\begin{array}{l}\text { Generalized anxiety } \\
\text { disorder (GAD) } \\
\text { Major depression } \\
\text { episode (MDE) }\end{array}$ & $\begin{array}{l}\text { Weak sense of coherence, } \\
\text { isolation, wrong beliefs, younger } \\
\text { age, threat perception (for oneself, } \\
\text { family, country, or world), } \\
\text { authorities mistrust, stigma, } \\
\text { financial problem, and too much } \\
\text { information about COVID- } \\
\text { 19COVID-19, especially from } \\
\text { social media and close social circle } \\
\text { such as family, friends, or coworker }\end{array}$ & $\begin{array}{l}\text { Strong sense of } \\
\text { coherence }\end{array}$ \\
\hline 4 & $\begin{array}{l}\text { Liang et al., } 2020 \\
\text { Guangdong, cross- } \\
\text { sectional, } \\
\text { prevalence study. }{ }^{7} \\
\text { JBI score } 8 / 9\end{array}$ & $\begin{array}{lr}4,164 & \text { college } \\
\text { students } & (48 \% \\
\text { female }) & \end{array}$ & $\begin{array}{l}\text { COVID-19 Fear Scale, } \\
\text { Patient Health } \\
\text { Questionnaire } \\
\text { Impact of Event Scale-6 }\end{array}$ & $\begin{array}{l}\text { Poor perceived mental health } \\
\text { status }\end{array}$ & $\begin{array}{l}\text { Monitor, intervention, } \\
\text { and coping strategy }\end{array}$ \\
\hline 5 & $\begin{array}{l}\text { Duan et al., } 2020 \\
\text { China, } \\
\text { Prevalence study, } \\
\text { Cross-sectional. }{ }^{8} \\
\text { JBI Score } 8 / 9\end{array}$ & $\begin{array}{l}1,390 \\
\text { participants } \\
(57.2 \% \text { female })\end{array}$ & $\begin{array}{lr}\text { Perceived } & \text { Stress Scale } \\
\text { Hostility } & \text { Subscale } \\
\text { Perceived } & \text { Social } \\
\text { Support Scale (PSSS) }\end{array}$ & $\begin{array}{l}\text { Young, financial problem, negative } \\
\text { perception }\end{array}$ & $\begin{array}{lr}\text { Social } & \text { support as } \\
\text { positive } & \text { coping } \\
\text { strategy } & \end{array}$ \\
\hline 6 & $\begin{array}{l}\text { Achraf et al., } 2020 \\
\text { Europe, North- } \\
\text { Africa, Western-Asia } \\
\text { and America, Cross- } \\
\text { sectional prevalence } \\
\text { study. }^{9} \\
\text { JBI Score } 8 / 9\end{array}$ & $\begin{array}{l}1,047 \\
\text { participants } \\
\text { (54\% women) }\end{array}$ & Depressive symptoms & $\begin{array}{l}\text { home confinement } \\
\text { depressive symptoms }\end{array}$ & $\begin{array}{l}\text { Active and Healthy } \\
\text { Confinement Lifestyle }\end{array}$ \\
\hline 7 & $\begin{array}{l}\text { Huang et al., } 2020 \\
\text { Hubei, Wuhan, } \\
\text { cross-sectional, } \\
\text { prevalence study. }{ }^{10} \\
\text { JBI Score } 7 / 9\end{array}$ & $\begin{array}{l}6261 \\
\text { participants } \\
(57.3 \% \text { female })\end{array}$ & $\begin{array}{l}\text { Perception of the } \\
\text { COVID-19 outbreak, } \\
\text { recent preventive or } \\
\text { avoidance behaviors, } \\
\text { and self-reported } \\
\text { mental health scales } \\
\text { including the Patient } \\
\text { Health Questionnaire } \\
\text { and Self-Rating Anxiety } \\
\text { Scale } \\
\end{array}$ & depression and anxiety & protective behaviors \\
\hline 8 & $\begin{array}{l}\text { Yan et al., } 2020 \\
\text { China, } \quad \text { cross- } \\
\text { sectional prevalence } \\
\text { study. }{ }^{11} \\
\text { JBI Score } 7 / 9\end{array}$ & $\begin{array}{l}3,233 \\
\text { participants } \\
\text { (54.4\% female) }\end{array}$ & $\begin{array}{l}\text { Stress scale and } \\
\text { boredom scale }\end{array}$ & Boredom, emotional distress & $\begin{array}{l}\text { Positive } \\
\text { strategies }\end{array}$ \\
\hline 9 & $\begin{array}{l}\text { Mahmud et al. } 2020 \text {, } \\
\text { Bangladesh, cross- } \\
\text { sectional, } \\
\text { prevalence study. }{ }^{12} \\
\text { JBI Score } 7 / 9\end{array}$ & $\begin{array}{l}246 \text { participants } \\
(45.3 \% \text { female })\end{array}$ & $\begin{array}{l}\text { Fear of COVID-19 } \\
\text { scale } \\
\text { Future career anxiety } \\
\text { Depression } \\
\text { COVID-19 }\end{array}$ & Depression and anxious & $\begin{array}{l}\text { Positive } \\
\text { strategies }\end{array}$ \\
\hline 10 & $\begin{array}{l}\text { Rogowska et al., } \\
2020, \text { Poland, } \\
\text { cross-sectional. }{ }^{13} \\
\text { JBI Score } 7 / 9\end{array}$ & $\begin{array}{l}914 \text { participants } \\
\text { (43.1\% female) }\end{array}$ & $\begin{array}{l}\text { Anxiety and Stress } \\
\text { Scale }\end{array}$ & $\begin{array}{l}\text { Female gender, negative coping } \\
\text { styles }\end{array}$ & Physical activity \\
\hline
\end{tabular}


Table 2. Characteristic, JBI score, measurement, and outcome of Inclusion Studies (continued)

\begin{tabular}{|c|c|c|c|c|c|}
\hline No & $\begin{array}{l}\text { Author, year, place, } \\
\text { study design, JBI } \\
\text { score }\end{array}$ & $\begin{array}{l}\text { Number of } \\
\text { participants, } \\
\text { demographic } \\
\text { characteristic }\end{array}$ & $\begin{array}{l}\text { Risk of psychiatric } \\
\text { problems } \\
\text { measurement } \\
\text { (score) }\end{array}$ & $\begin{array}{l}\text { Negative psychosocial } \\
\text { impacts/attitude/less favorable } \\
\text { demographic characteristics }\end{array}$ & $\begin{array}{l}\text { Positive protective } \\
\text { factors } \\
\text { attitude/favorable } \\
\text { demographic } \\
\text { characteristics }\end{array}$ \\
\hline 11 & $\begin{array}{l}\text { Duplaga } \\
\text { Gryztar, } 2020 \\
\text { Poland, and } \\
\text { sectional, } \\
\text { prevalence study. }{ }^{14} \\
\text { JBI score 6/9 }\end{array}$ & $\begin{array}{l}1,002 \\
\text { participants } \\
(50.6 \% \text { female })\end{array}$ & $\begin{array}{l}\text { Health Questionnaire } \\
\text { eHealth Literacy } \\
\text { Scale }\end{array}$ & Female, entrepreneurs or farmers & $\begin{array}{l}\text { Individual motivation, } \\
\text { willing to adopt }\end{array}$ \\
\hline 12 & $\begin{array}{l}\text { Joseph et al., } 2020 \\
\text { Saudi Arabia, cross- } \\
\text { sectional, } \\
\text { prevalence study. }{ }^{15} \\
\text { JBI Score } 7 / 9\end{array}$ & $\begin{array}{l}584 \text { participants } \\
(31.8 \% \text { female })\end{array}$ & $\begin{array}{l}\text { Depression } \\
\text { symptoms }\end{array}$ & $\begin{array}{l}\text { Female, had a history of mental } \\
\text { illness }\end{array}$ & $\begin{array}{l}\text { Early detection and } \\
\text { treatment }\end{array}$ \\
\hline 13 & $\begin{array}{l}\text { Gascon et al., } 2020 \text {, } \\
\text { Spain, cross- } \\
\text { sectional, } \\
\text { prevalence study. }{ }^{16} \\
\text { JBI Score } 6 / 9\end{array}$ & $\begin{array}{l}174 \text { participants } \\
(56 \% \text { female })\end{array}$ & $\begin{array}{l}\text { Pseudoscientific } \\
\text { beliefs }\end{array}$ & $\begin{array}{l}\text { Pseudoscientific beliefs, } \\
\text { realization,depersonalization, } \\
\text { paranoid perceptions }\end{array}$ & $\begin{array}{l}\text { Beware of the } \\
\text { information truth }\end{array}$ \\
\hline 14 & $\begin{array}{l}\text { Miranda et al., } 2020 \\
\text { Columbia, cross- } \\
\text { sectional analytics. }{ }^{17} \\
\text { JBI Score } 5 / 8\end{array}$ & $\begin{array}{l}1,687 \\
\text { participants }(59 \% \\
\text { female) }\end{array}$ & $\begin{array}{l}\text { Stigma Discrimination } \\
\text { The COVID-5 Fear } \\
\text { Scale }\end{array}$ & fear of COVID-19, stigma & $\begin{array}{l}\text { Effective cross cultural } \\
\text { intervention }\end{array}$ \\
\hline 15 & $\begin{array}{l}\text { Bakioglu et al., 2020, } \\
\text { Turkey, cross- } \\
\text { sectional, } \\
\text { prevalence study. }{ }^{18} \\
\text { JBI score } 7 / 9\end{array}$ & $\begin{array}{l}960 \text { participants } \\
(69 \% \text { female })\end{array}$ & $\begin{array}{l}\text { The Fear of COVID- } \\
19 \text { Scale (FCV-19s) } \\
\text { The Positivity Scale } \\
\text { The Intolerance of } \\
\text { Uncertainty Scale } \\
\text { (IUS-12), Depression, } \\
\text { Anxiety, and Stress } \\
\text { Scale (DAS-21) }\end{array}$ & $\begin{array}{l}\text { female, had chronic disease, } \\
\text { elderly, intolerance of uncertainty, } \\
\text { depression, anxiety, and stress are } \\
\text { mediating role }\end{array}$ & $\begin{array}{lr}\text { Spend time in safe } \\
\text { environment, } \\
\text { performing } & \text { family } \\
\text { tasks, relaxing, doing } \\
\text { hobbies }\end{array}$ \\
\hline 16 & $\begin{array}{l}\text { Damirchi et al., } 2020 \\
\text { Iran, cross-sectional, } \\
\text { descriptive and } \\
\text { correlational study. }{ }^{19} \\
\text { JBI Score } 6 / 9\end{array}$ & $\begin{array}{l}354 \text { participants } \\
(42.3 \% \text { female })\end{array}$ & $\begin{array}{l}\text { Self-Talk } \\
\text { questionnaires, } \\
\text { Coping Strategies } \\
\text { questionnaires }\end{array}$ & $\begin{array}{l}\text { negative self-talk, negative coping } \\
\text { style }\end{array}$ & $\begin{array}{l}\text { self-talk predicted } \\
\text { problem centered style }\end{array}$ \\
\hline 17 & $\begin{array}{l}\text { Wahlund et al., } 2020 \\
\text { Sweden, } \\
\text { randomized control } \\
\text { trial. }{ }^{20} \\
\text { JBI Score } 11 / 13\end{array}$ & $\begin{array}{l}670 \text { participants } \\
(83 \% \text { female })\end{array}$ & $\begin{array}{l}\text { Anxiety scale } \\
\text { administered at } \\
\text { baseline and weeks } \\
1-3 \text { Treatment was } \\
\text { cognitive behavioral } \\
\text { therapy }\end{array}$ & Anxious, worry & $\begin{array}{l}\text { Self-guided } \\
\text { psychological } \\
\text { intervention }\end{array}$ \\
\hline 18 & $\begin{array}{l}\text { Song et al., } 2020 \\
\text { China, } \\
\text { sectional, } \\
\text { prevalence study. } \\
\text { JBI Score } 7 / 9\end{array}$ & $\begin{array}{l}3,180 \\
\text { participants } \\
(72.1 \% \text { female })\end{array}$ & $\begin{array}{lr}\text { Anxiety Scale } & \\
\text { Depression } & \text { Scale } \\
\text { (SDS) } & \text { Simplified } \\
\text { Coping } & \text { Style } \\
\text { Questionnaire } & \\
\end{array}$ & $\begin{array}{l}\text { Female, younger, less education, } \\
\text { unmarried }\end{array}$ & $\begin{array}{l}\text { Resilience, } \\
\text { coping style }\end{array}$ \\
\hline 19 & $\begin{array}{l}\text { Cansel et al., } 2020 \\
\text { Turkey, } \quad \text { cross- } \\
\text { sectional. }{ }^{22} \\
\text { JBI Score } 7 / 9\end{array}$ & $\begin{array}{l}3,549 \\
\text { participants } \\
(60.9 \% \text { female })\end{array}$ & $\begin{array}{l}\text { Depression, Anxiety } \\
\text { and Stress Scale }\end{array}$ & $\begin{array}{l}\text { Female, young age, higher } \\
\text { education levels }\end{array}$ & $\begin{array}{l}\text { Compliance with the } \\
\text { rules }\end{array}$ \\
\hline
\end{tabular}

It increased more anxiety and depression. Sometimes it was called infodemic. ${ }^{11}$ Infodemic can cause wrong beliefs and threat perception for oneself, family, country, or world. ${ }^{2}$

A weak sense of coherence, isolation, stigma, and financial problem at a long time during the COVID-19 pandemic can cause anxiety and depression. ${ }^{2}$ Lowerincome and jobless patients increased their level of perceived stress. Isolation and quarantine escalated depression risk. ${ }^{8}$ Feeling insecure about the future career had a negative effect on human psychology. The policymaker had to make a special plan to boost up economic growth. ${ }^{12}$

People who had family members or friends getting infected with COVID-19 reported a greater score of anxiety. Therefore, providing truthful information is a must. The perception that COVID-19 can be controlled by protective behavior is related to the lower anxiety scale. ${ }^{10}$

\section{Positive risk factors (protective factors) Positive demographic} characteristics for decreasing the risk of 
psychiatric problems during the COVID-19 pandemic were single and young. Younger age has a lower risk of having severe COVID-19 infection. That's why the depression and anxiety score was lower among them. ${ }^{8}$ However, Schnell and Krampe showed that less acute COVID-19 stress was lower for older age, married people, and active workers. This might be due to less outdoor activity is needed to be done by the elderly. They can stay at home to reduce the risk of COVID-19 infection. ${ }^{6}$

Positive attitudes for decreasing the risk of psychiatric problems during the COVID-19 pandemic are enhancing spirituality and having good knowledge about COVID-19. Good knowledge means there must be an optimal knowledge. Too much information will lead to anxiety and depression. ${ }^{5}$ A strong sense of coherence is also a good protective factor to decrease the risk of depression and generalized anxiety disorder. ${ }^{2}$

Positive coping was associated with a healthier psychological state. A positive coping role is a resilient factor. It is including an adaptive mechanism. Boredom normally happened during isolation and work from home. However, mindfulness training at home can be done as a positive activity. Optimal and proper usage of social media can increase positive coping behaviors. ${ }^{11}$ Individual motivation and willingness to adapt to any situation can help in increasing health literacy. ${ }^{14}$

College students also encountered anxiety during the COVID-19 pandemic. They have to study at home. ${ }^{13}$ Home confinement can cause depressive symptoms. Monitoring their psychological status is very essential. Coping strategy can be done through seeking psychological help. ${ }^{7}$ Social support is very helpful during this pandemic. $^{8}$ Active and Health Confinement Lifestyle had to be implemented. ${ }^{9} \quad$ Physical activity, satisfaction with life, and task-oriented coping can be done to reduce anxiety. ${ }^{13}$ Relaxing and doing hobbies, including spending time in a safe environment with family may decrease the fear to COVID$19 .{ }^{18}$

Early detection and treatment are important to prevent any progression from depression to suicide. ${ }^{15}$ Pseudoscientific beliefs are very dangerous. It contains derealization and depersonalization. Paranoids can develop from excessive information. Ensure information credibility and analyze the information before sharing can prevent misinformation. ${ }^{16}$

Stigma discrimination can develop when someone got COVID-19 infection. This stigma increases the fear of COVID19. Effective cross-cultural communication is a strategy to prevent stigma. ${ }^{17}$ Positive self-talk has a positive relationship with problem-center coping style. It can reduce stigma and depression. ${ }^{19}$ Compliance with the rule is one way to reduce the possibility of infection and anxiety. ${ }^{22}$

\section{Conclusion}

Anxiety and depression prevention related to Covid-19 can be implemented through strengthening the positive protective factor and minimize the risk factors. Positive protective factors are positive attitude and coping mechanisms towards COVID-19 pandemic. Positive mental strengthening activities can promote good mental health. Therefore, the COVID19 pandemic should be seen as an opportunity to strengthen the positive mental attitudes.

\section{Acknowledgment}

None

\section{Funding \\ None}

\section{Author Contribution}

The author is only one, therefore all manuscript was written by one people.

\section{Ethics approval}

None (this is a systematic review)

\section{References}

1. Li WW, $\mathrm{Yu} \mathrm{H}$, Miller DJ, Yang $\mathrm{F}$, Rouen C. Novelty Seeking and Mental Health in Chinese University Students Before, During, and After the COVID-19 Pandemic Lockdown: A Longitudinal Study. Front Psychiatry. 2020;11(December):115.

2. Genereux M, Schluter PJ, Hung 
KKC, Wong CS, Pui C, Mok Y, et al. One Virus, Four Continents, Eight Countries: An Interdisciplinary and International Study on the Psychosocial Impacts of the COVID19 Pandemic among Adults. Int J Environ Res Public Health. 2020;17(8390):1-16.

3. Montag C, Elhai JD. Addictive Behaviors Reports Discussing digital technology overuse in children and adolescents during the COVID-19 pandemic and beyond: On the importance of considering Affective Neuroscience Theory. Addict Behav Reports [Internet]. 2020;12:100313. Available from: https://doi.org/10.1016/j.abrep.2020. 100313

4. Rakovac A, Andric L, Karan V, Bogdan M, Slavic D, Klasnja A. Evaluation of spirometric parameters and maximum oxygen consumption in athletes and non-athletes. Med Pregl Rev. 2018;71(5-6):157-61.

5. Rias YA, Rosyad YS, Chipojola R, Wiratama BS. Effects of Spirituality, Knowledge, Attitudes, and Practices toward Anxiety Regarding COVID-19 among the General Population in INDONESIA: A Cross-Sectional Study. J Clin Med. 2020;9(3798):116.

6. Schnell T, Krampe H. Meaning in Life and Self-Control Buffer Stress in Times of COVID-19: Moderating and Mediating Effects With Regard to Mental Distress. Front Psychiatry. 2020;11(September):1-16.

7. $\quad$ Liang S, Chen R, Liu L, Li X, Chen J. The Psychological Impact of the COVID-19 Epidemic on Guangdong College Students: The Difference Between Seeking and Not Seeking Psychological Help. Front Psychol. 2020;11(September):1-10.

8. Duan H, Yan L, Ding X, Gan Y, Kohn $\mathrm{N}, \mathrm{Wu}$ J. Impact of the COVID-19 pandemic on mental health in the general Chinese population: Changes, predictors and psychosocial correlates. Psychiatry Res. 2020;293(2020):113396.

9. Achraf $A$, Mueller $P$, Trabelsi $K$, Chtourou H, Boukhris O, Masmoudi
L, et al. PLOS ONE Psychological consequences of COVID-19 home confinement: The ECLB-COVID19 multicenter study. PLoS One. 2020; (November):1-13.

10. Huang J, Liu F, Teng Z, Chen J, Zhao $J$, Wang $X$, et al. Public Behavior Change, Perceptions, Depression, and Anxiety in Relation to the COVID19 Outbreak. Open Forum Infect Dis. 2020;1-8.

11. Yan L, Gan $Y$, Ding $X$, Wu J, Duan $H$. The relationship between perceived stress and emotional distress during the COVID-19 outbreak: Effects of boredom proneness and coping style. J Anxiety Disord. 2021;77(2021):102328.

12. Mahmud S, Talukder MU, Rahman SM. Does 'Fear of COVID-19' trigger future career anxiety? An empirical investigation considering depression from COVID-19 as a mediator. Int J Soc Psychiatry. 2020;1-11.

13. Rogowska A, Kuśnierz C, Bokszczanin A. Examining Anxiety, Life Satisfaction, General Health , Stress and Coping Styles During COVID-19 Pandemic in Polish Sample of University Students. Psychol Res Behav Manag. 2020;13:797-811.

14. Duplaga $M$, Grysztar $M$. The Association between Future Anxiety , Health Literacy and the Perception of the COVID-19 Pandemic: A CrossSectional Study. Healthcare. 2021;9(43).

15. Joseph R, Lucca JM, Alshayban D, Alshehry YA. The immediate psychological response of the general population in Saudi Arabia during COVID-19 pandemic: A crosssectional study. J Infect Public Health. 2021;14:1-8.

16. Escolà-gascón Á, Marín F, Rusiñol J, Gallifa J. Pseudoscientific beliefs and psychopathological risks increase after COVID-19 social quarantine. Global Health. 2020;16(72):1-11.

17. Cassiani-miranda CA, Campo-arias, Tirado-otálvaro AF, Botero-tobón LA, Upegui-arango LD, Rodríguezverdugo MS, et al. Stigmatisation associated with COVID-19 in the 
general Colombian population. Int $\mathrm{J}$ Soc Psychiatry. 2020;1-9.

18. Bakioglu F, Korkmaz O, Ercan $\mathrm{H}$. Fear of COVID-19 and Positivity: Mediating Role of Intolerance of Uncertainty, Depression, Anxiety , and Stress. Int J Ment Health Addict. 2020;1-14.

19. Damirchi ES, Mojarrad A, Pireinaladin S, Grjibovski AM. The Role of Self-Talk in Predicting Death Anxiety , Obsessive-Compulsive Disorder, and Coping Strategies in the Face of Coronavirus Disease ( COVID-19 ). Iran J Psychiatry. 2020;15(3):182-8.

20. Wahlund T, Klara M, Lauri O. Brief
Online Cognitive Behavioural Intervention for Dysfunctional Worry Related to the COVID-19 Pandemic: A Randomised Controlled Trial. Psychother Psychosom. 2020;1-9.

21. Song $S$, Yang $X$, Yang $H$, Zhou $P, M a$ $H$, Teng C. Psychological Resilience as a Protective Factor for Depression and Anxiety Among the Public During the Outbreak of COVID-19. Front Psychol. 2021;11:1-8.

22. Cansel N, Ucuz I, Kadir A, Burcu A, Tetik K, Colak C, et al. Prevalence and predictors of psychological response during immediate COVID19 pandemic. Int J Clin Pract. 2021; (January):1-14. 